\title{
A MEM Vibratory Gyro with Mode-Matching Achieved by Resonator Mass Loading
}

\author{
Dennis Kim* and Robert M'Closkey ${ }^{\dagger}$ \\ Mechanical and Aerospace Engineering Department \\ Henry Samueli School of Engineering and Applied Science \\ University of California, Los Angeles
}

\begin{abstract}
This paper presents preliminary performance results for a vibratory rate gyro based on a novel MEM resonator whose design facilitates frequency mismatch reduction via mass loading. A model-based systematic tuning algorithm was implemented to reduce the $n=2$ modal frequency mismatch without impacting the quality factors. Mass loading the resonator eliminates the need for electrostatic tuning and only a single bias voltage to the resonator is required for operation as a rate sensor. Force-to-rebalance operation with a $10 \mathrm{~Hz}$ bandwidth yielded a minimum rate bias instability of $0.11 \mathrm{deg} / \mathrm{hr}$ and an estimated angle random walk of $0.02 \mathrm{deg} /$ root-hr. A decrease in rate noise was also demonstrated as the sensor's cross-channel coupling was reduced by aligning the forcer/pick-off frame with the anti-nodes of the modes.
\end{abstract}

Index Terms - vibratory gyro, mode matching, frequency mismatch

\section{INTRODUCTION}

Vibratory rate gyros exploit the Coriolis-coupled modes of a mechanical resonator for angular rate detection. Modal frequency matching or "tuning" is essential to achieve the sensor's optimal performance when buffer electrical noise is the dominant noise source. For high quality factor devices, the sensor's angle random walk (ARW) will degrade in proportion to the degree of detuning if the detuning exceeds the resonator's intrinsic mechanical bandwidth [1]. For example, a mere $1 \mathrm{~Hz}$ frequency mismatch in a resonator whose nominal resonant frequencies and quality factors are $15 \mathrm{kHz}$ and $75 \mathrm{~K}$, respectively, will increase the ARW by an order of magnitude. In MEM gyros, tuning is typically achieved by biasing dedicated electrodes so as to create electrostatic "springs" to modify the resonator's stiffness. Although electrostatic modal frequency tuning has been most widely used and successfully applied to MEM gyros [2]-[5], this complicates the sensor electronics because the biases must be held to high degree of stability, especially with regard to temperature variations. The sensor's quadrature signal can be quite sensitive to the tuning bias voltages so small demodulation phase errors will introduce a (possibly time varying) term into the estimated rate signal. Elimination of electrostatic tuning biases not only greatly simplifies the sensor electronics but also removes a source of rate

* Email: dongj@seas.ucla.edu

† Corresponding author. Email: rtm@seas.ucla.edu bias instability. An alternative tuning approach in lieu of modifying the resonator's stiffness is to perturb the mass distribution of the resonator. In order to demonstrate modal frequency tuning techniques on MEM resonators, a resonator was designed and fabricated to accommodate mass loading as opposed to mass removal, mainly to avoid damage to the resonator that can occur during ablation. We have successfully implemented a systematic mass tuning algorithm using point mass perturbations so that the frequency mismatch of the resonator is reduced to less than the resonator's open-loop bandwidth [6]. In this paper, we present a case study for a resonator whose frequency mismatch of the $n=2$ Coriolis coupled modes was reduced from $14.2 \mathrm{~Hz}$ to $0.07 \mathrm{~Hz}$ using point mass perturbations. No electrical biasing was used for tuning the modal frequencies and the mass loading permanently modified the resonator dynamics. The tuned resonator was then configured for force-to-rebalance angular rate sensing mode to characterize its noise performance as a rate gyro.

The paper is organized as follows. Section II introduces the resonator design and briefly describes the mass loading techniques. Experimental results with a tuned resonator are reported in Section III. A second tuned resonator is used to demonstrate the reduction in the rate bias noise as the crosschannel coupling in the open-loop resonator is decreased. Section IV concludes the paper.

\section{Resonator Design}

The resonator is $10 \mathrm{~mm}$ in diameter with a number of thin concentric rings connected by alternating spokes as shown in Fig. 1(a). The central post of the resonator is bonded to the baseplate so that the rings can vibrate freely in the plane. The resonator is surrounded by 24 electrodes which are used for electrostatic actuation and capacitive sensing with a $20 \mu \mathrm{m}$ gap. Four additional flag-shaped pads are connected to the resonator for DC biasing (Fig. 1(a)). For $\mathrm{n}=2$ modal pair testing, three neighboring electrode segments are combined to render a larger electrode area for each of two sensing pick-offs and two forcers, labelled as $s_{1}, s_{2}$ and $d_{1}, d_{2}$, respectively. The sensing electrodes are physically oriented at 45deg to each other and their companion driving electrodes are assigned on the opposite side of the resonator. The rest 


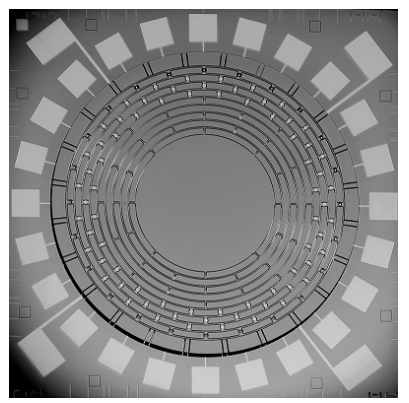

(a) Resonator and electrodes

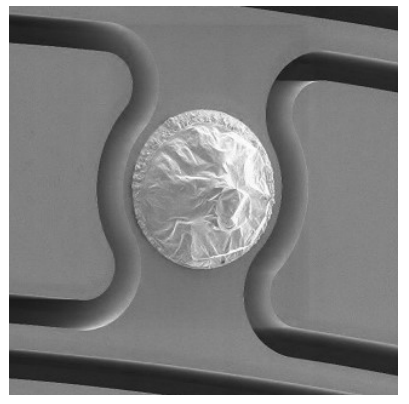

(c) Reflowed solder sphere

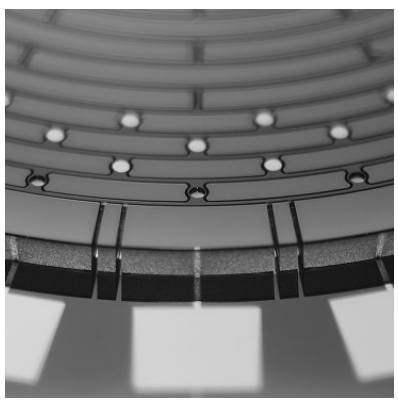

(b) Reservoirs and gold pads

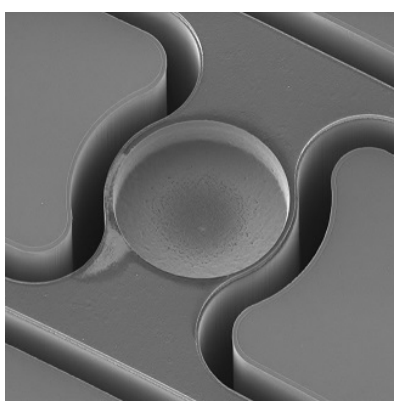

(d) Silver nano-particle ink
Fig. 1. Photos of the resonator and mass perturbations. (a) A $10 \mathrm{~mm}$ diameter resonator bonded to the base plate is surrounded by 24 external electrodes. Each external electrode is electrically connected to a squareshaped pad and the resonator is connected to the four flag-shaped pads for voltage biasing. (b) The reservoirs are etched into the resonator's outer most ring and the $130 \mu \mathrm{m}$ diameter gold pads are located in the inner rings. (c) A single precision solder sphere is reflowed onto the gold pad to provide a coarse mass perturbation. (d) Multiple silver nano-particle ink droplets are deposited into the reservoir for fine mass perturbations.

of the external electrodes are connected to the resonator bias. The $n=2$ modal frequencies are near $13 \mathrm{kHz}$ and have approximately $50 \mathrm{~K}$ quality factors. To take full advantage of these high quality factors, the frequency mismatch should be reduced to a level comparable to open-loop bandwidth of the modes, which in these resonators is approximately $200 \mathrm{mHz}$. Since the as-fabricated $n=2$ frequency mismatches almost always exceed the open-loop bandwidth, frequency mismatch reduction is necessary for optimum gyro performance. There are a total of 96 locations on the resonator (Fig. 1(b)) where mass loading can be performed by reflowing $75 \mu \mathrm{m}$ diameter precision solder spheres onto $130 \mu \mathrm{m}$ diameter gold pads (Fig. 1(c)) or depositing $40 \mu \mathrm{m}$ diameter silver nanoparticle ink droplets into reservoirs etched into the resonator (Fig. 1(d)). The solder sphere provides a very uniform mass quanta and is used for coarse tuning of the resonator dynamics and the silver ink allows fine tuning capability with a small perturbation provided by a single droplet. The mass tuning approach has been successfully implemented on a number of these resonators with the final $n=2$ mismatch reduced to less than $100 \mathrm{mHz}$ even when the initial frequency mismatch exceeds $30 \mathrm{~Hz}$. More details on the mass tuning process will be reported elsewhere.
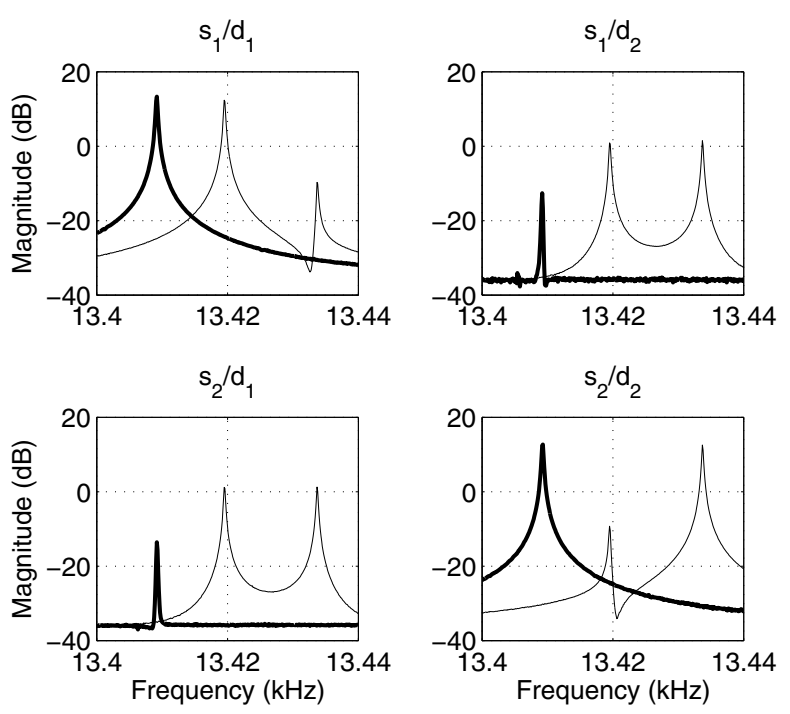

Fig. 2. Empirical frequency response of the resonator. Two-input/twooutput magnitude plots in its "untuned" native state (thin) clearly show two distinctive modes with a $14.2 \mathrm{~Hz}$ frequency mismatch. The tuned resonator (thick) displays a single peak in each channel and the analytical model fit to the data suggests a $0.07 \mathrm{~Hz}$ frequency mismatch. Note that the inputs $\left(d_{1}, d_{2}\right)$ and outputs $\left(s_{1}, s_{2}\right)$ are in volts. The parasitic coupling between forcing and sensing electrodes accounts for the flat level in each plot.

\section{EXPERIMENTAL RESULTS}

\section{A. Open-loop resonator dynamics}

All tests were performed with the resonator inside a vacuum chamber whose pressure was maintained below $1 \mathrm{~m}$ Torr. A DC potential of 40volts is used to bias the resonator and to capture the open-loop resonator dynamics. An input signal is directly applied to a forcing electrode to create a radial electrostatic force to excite the resonator. Each sensing electrode is connected to the virtual ground of a transresistance amplifier so that the in-plane motion of the resonator is converted into a buffered output voltage that is proportional to the radial velocity of the resonator averaged over the electrode area. Fig. 2 compares the empirical frequency response data of the open-loop resonator dynamics before and after mass loading. The initial resonator dynamics prior to any mass perturbation reveals the frequency mismatch of $14.7 \mathrm{~Hz}$ and at the termination of the mass perturbation the modal frequencies are very close since only a single mode is evident in each channel. An identified parametric model indicates a frequency mismatch of $0.07 \mathrm{~Hz}$.

\section{B. Force-to-rebalance operation}

The tuned resonator was configured for force-to-rebalance operation in which a self-exciting automatic gain control (AGC) maintained a stable sinusoidal response of $s_{1}$ at the tuned modal frequency and a second feedback loop nulled $s_{2}$ signal. The angular rate is inferred from the feedback signal. More details of the control architecture used for this type of resonator may be found in [7]. To operate the sensor, only a single resonator bias voltage was required to facilitate the sensing and actuation. The force-to-rebalance loop was 


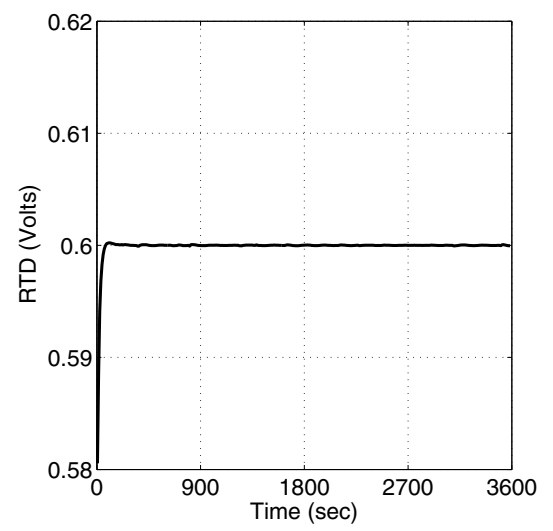

(a) RTD measurements

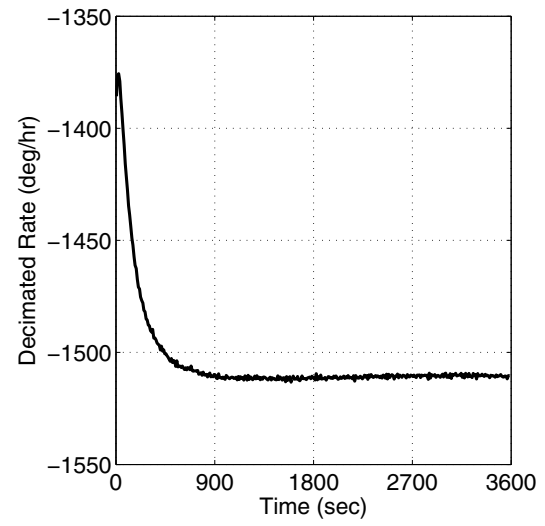

(b) Decimated zero-rate bias

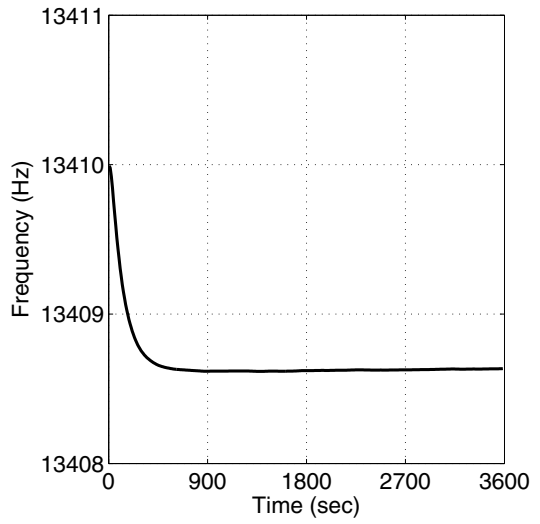

(c) Sensor's operating frequency

Fig. 3. (a) Voltage measurement of RTD trace was regulated with the heating trace of thermal platform. (b) The initial transient response in zero-rate bias data can be attributed to the resonator's temperature change. (c) The sensor's operating frequency shows a very similar trend as the zero-rate bias. The steady-state RTD and operating frequency measurements estimate the resonator temperature of $33.5^{\circ} \mathrm{C}$ when the resonator is thermally stabilized. The time domain data from $900 \mathrm{sec}$ to $3600 \mathrm{sec}$ are processed to generate the Allan variance as well as the power spectral density plots shown in Fig. 4 and Fig. 5, respectively.

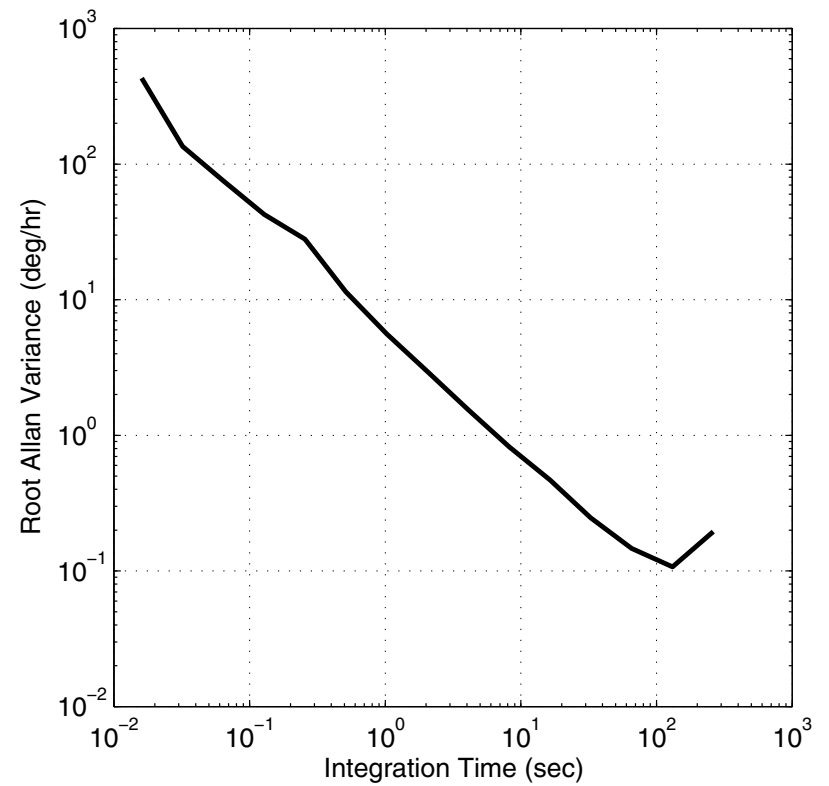

Fig. 4. The square root of the Allan variance. The minimum rate bias instability is $0.11 \mathrm{deg} / \mathrm{hr}$ at about $100 \mathrm{sec}$ integration time and the Allan variance increases at longer integration times due to a slow drift in the zero-rate bias.

adjusted to achieve a $10 \mathrm{~Hz}$ bandwidth. The vacuum chamber is located on a limited motion turn-table and permits the measurement of the scale factor to within a few percent. Temperature-induced drift is minimized by bonding the resonator die to a $12 \mathrm{~mm}$ by $18 \mathrm{~mm}$ thermal platform. The platform has two separate platinum traces, one of which is used for heating and the other for resistance temperature detector (RTD)-based temperature sensing. Fig. 3 plots decimated $(1 \mathrm{~Hz})$ time response data of the regulated RTD

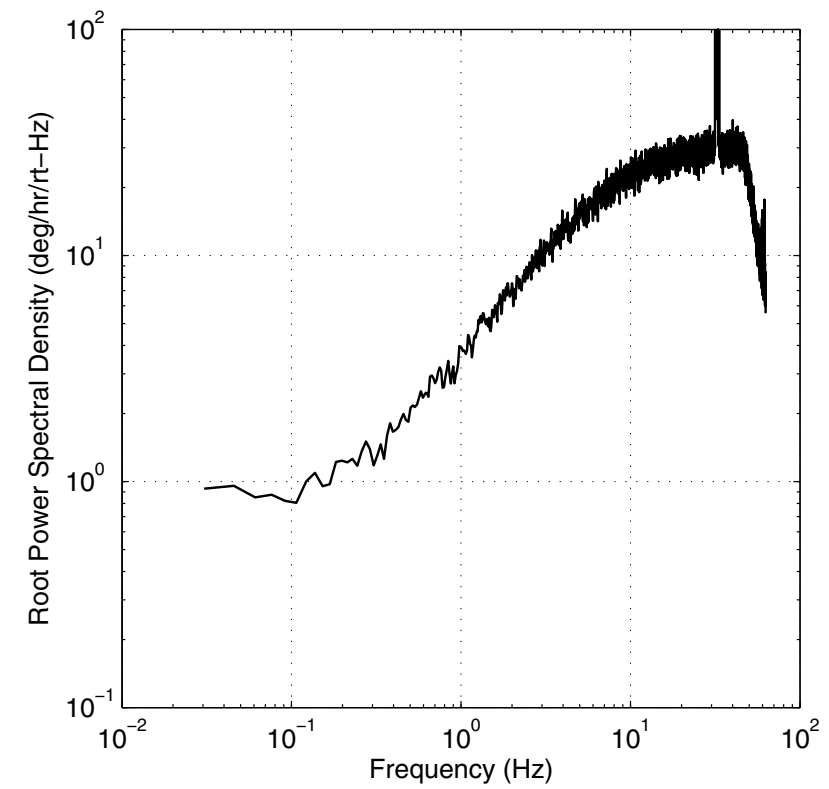

Fig. 5. The square root of rate equivalent power spectral density. The low frequency asymptote suggests the angle random walk, $0.02 \mathrm{deg} / \mathrm{hr} / \mathrm{rt}-\mathrm{hr}$. The high frequency corner at $10 \mathrm{~Hz}$ represents the sensor's measurement bandwidth and the roll-off at $62.5 \mathrm{~Hz}$ corresponds to the corner frequency of the anti-aliasing filter.

voltage, zero-rate bias, and sensor's operating frequency. Although the heater/RTD temperature regulation loop stabilizes rapidly (Fig. 3(a)), the zero-rate bias displays a slower transient response in Fig. 3(b). The sensor's operating frequency (Fig. 3(c)) is strongly correlated with the resonator temperature. The difference in thermal time constants may be attributed to the fact that the resonator die and the thermal platform are not collocated. The measured zero-rate bias 

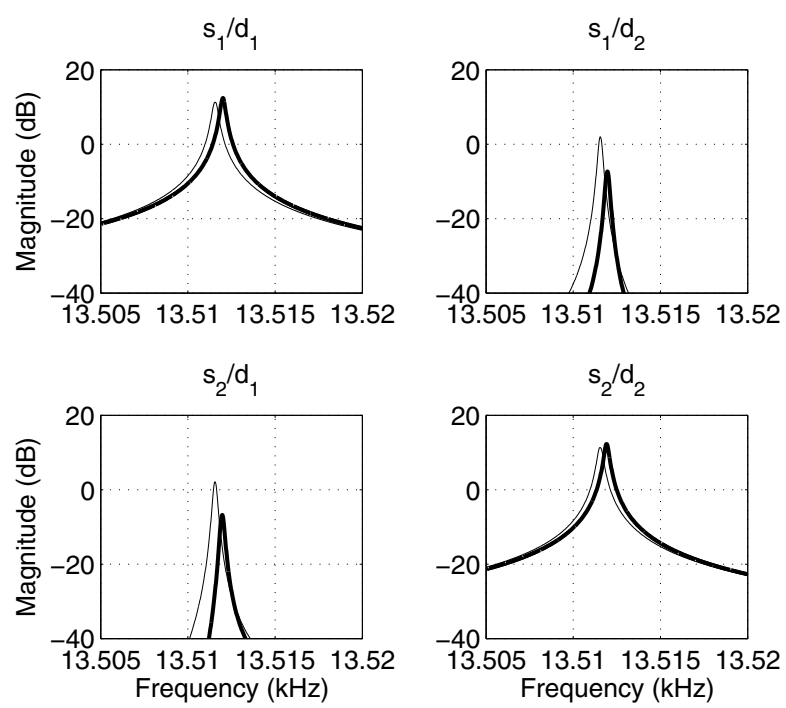

Fig. 6. Frequency response of the identified model. The original tuned dynamics (thin) displays higher cross-channel gains than the re-aligned tuned dynamics (thick) although the frequency mismatch and quality factors are invariant.

data from $900 \mathrm{sec}$ to $3600 \mathrm{sec}$ yielded a minimum rate bias instability of $0.11 \mathrm{deg} / \mathrm{hr}$ at $100 \mathrm{sec}$ from the standard Allan deviation plot (see Fig. 4). The ARW is estimated to be $0.02 \mathrm{deg} /$ root-hr from the power spectral density plot in Fig. 5 (the ARW corresponds to the low frequency asymptote).

\section{Effect of cross-channel coupling}

A second resonator was used to determine the impact on gyro performance of cross-channel coupling in the resonator dynamics. The cross-channel coupling is given by the offdiagonal channels of the frequency response data in Figs. 2 and 6 and quantifies how the AGC loop forcer signal couples into the force-to-rebalance loop. The cross-channel coupling cannot be removed by either electrostatic tuning or mass loading alone because the former modifies the resonator stiffness matrix to be a scaled version of the mass matrix (in general, non-diagonal) and the latter modifies the resonator mass matrix to be a scaled copy of the stiffness matrix (also in general, non-diagonal). Either approach yields, in principle, a frequency mismatch of zero, however, the mass and stiffness matrices still retain a pair of unique eigenvectors which define the anti-nodes of the $n=2$ modes. If the sensing and actuation electrodes are not optimally aligned with respect to the anti-nodes, cross-coupling between the excitation and force-to-rebalance channels is created. The sinusoidal component of the AGC forcer signal creates, through this coupling, non-zero biases in the demodulated rate and quadrature signals. Any change in the coupling term will create drift in these bias signals, however, even if the drift is eliminated the coupling still has a secondary effect of introducing noise into the force-to-rebalance loop from the AGC forcer signal. For example, AGC forcer noise can be created by DAC quantization that, in general, cannot be rejected with phase-sensitive demodulation.

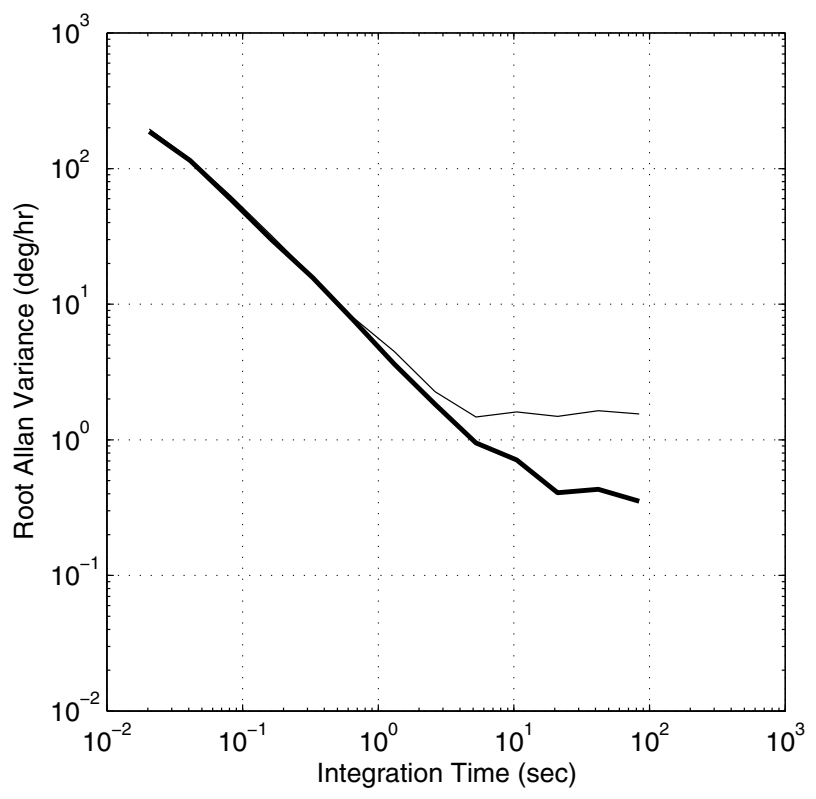

Fig. 7. The square root of the Allan variance. When the resonator displayed a higher cross-channel coupling, the minimum rate bias instability was limited to $1.5 \mathrm{deg} / \mathrm{hr}$ (thin). After the cross-channel coupling was reduced by aligning the forcer/pick-off frame with the anti-nodes frame, the minimum rate bias instability improved to $0.4 \mathrm{deg} / \mathrm{hr}$ (thick).

The flexibility of the resonator permits the reassignment and grouping of the electrodes after tuning in order to better align the forcer/pick-off frame with the anti-nodes of the modes. The second resonator's frequency mismatch was reduced to less than $0.1 \mathrm{~Hz}$ and Fig. 6 compares the resonator dynamics before and after aligning the forcer/pick-off frame with the anti-nodes of the frequency-matched modes. While the diagonal channel gains and the frequency mismatch are comparable, the off-diagonal channel gains are reduced from $2 \mathrm{~dB}$ to $-7 \mathrm{~dB}$ after the re-alignment. As the crosschannel gain is reduced, the improvement of sensor's noise performance under the same operating conditions is quite evident in the standard Allan variance plots in Fig. 7. For both cases, the zero-rate bias data were detrended to remove the effect of long term drift due to changes in the resonator dynamics. Whereas the minimum rate bias instability was limited to $1.5 \mathrm{deg} / \mathrm{hr}$ for the resonator with the original electrode configuration, the re-aligned electrodes achieved an improved minimum rate bias instability of $0.4 \mathrm{deg} / \mathrm{hr}$.

\section{CONCLUSION}

We have presented performance results of a vibratory rate gyro with a mass tuned MEM resonator. The frequency matching was essential to achieve the sensor's ultimate performance and the tuning via mass loading allowed the sensor to operate with a single voltage bias. The current $0.11 \mathrm{deg} / \mathrm{hr}$ rate bias instability and $0.02 \mathrm{deg} /$ root-hr ARW may be further improved by providing better environmental control as well as increasing the sensor electrode area. Experiments and 
modeling of the cross-channel coupling and its impact on the rate noise are ongoing and will be reported at a future date.

\section{ACKNOWLEDGEMENTS}

The authors thank the Defense Advanced Research Projects Agency (DARPA) micro-PNT program for financial support under contract W31P4Q-11-1-0004. The authors also thank Jenny Kim for taking high quality photos of the resonator.

\section{REFERENCES}

[1] D. Kim and R.T. MCloskey, "Spectral analysis of vibratory gyro noise, IEEE Sensors Journal, Vol. 13, No. 11, pp. 4361-4374, Nov. 2013.

[2] D. Keymeulen, W. Fink, M. Ferguson, C. Peay, B. Oks, R. Terrile, and K. Yee, "Tuning of MEMS devices using evolutionary computation and open-loop frequency response," in IEEE Aerospace Conf., 2005, pp. $1-8$.

[3] D. Kim and R.T. M'Closkey, "A systematic method for tuning the dynamics of electrostatically actuated vibratory gyros," IEEE Trans. Control System Technology, Vol. 14, No. 1, Jan. 2006, pp. 69-81.

[4] A. Sharma, M. Zaman, and F. Ayazi," "A sub-0.2deg/hr bias drift micromechanical silicon gyroscope with automatic CMOS modematching," in IEEE Journal of Solid-State Circuits,, Vol. 44, No. 5, May 2009, pp. 1593-1608.

[5] I. Prikhodko, S. Zotov, A. Trusov, and A. Shkel, "Foucault pendulum on a chip: rate integrating silicon MEMS gyroscope," in Sensors and Actuators A: Physical, Vol. 177, Apr. 2012, pp. 67-78.

[6] D. Schwartz, D. Kim, and R.T. M'Closkey, "A model-based approach to multi-modal mass tuning of a micro-scale resonator," in Proc. Amer. Control Conf., 2012, pp. 98-103.

[7] D. Kim and R.T. MCloskey, "Dissecting tuned MEMS vibratory gyros, in Feedback Control of MEMS to Atoms, J. J. Gorman and B. Shapiro, Eds. Springer, 2012. 